\title{
EXPECTATIONS OVER DETERMINISTIC FRACTAL SETS
}

\author{
MICHAEL G. ROSE
}

(Received 10 November 2015; first published online 25 April 2016)

2010 Mathematics subject classification: primary 28A80; secondary 28A25, 37A30, 37M25, 41A55, 65-04.

Keywords and phrases: fractal, iterated function system, box integral, ergodic theory.

Motivated by the need for new mathematical tools applicable to the analysis of fractal point-cloud distributions, this thesis presents a measure-theoretic foundation for the consideration of expectations of smooth complex-valued functions over deterministic fractal domains.

Initial development of the theory of fractal expectations proceeds from extension of the classical theory of box integrals (pertaining to separation moments over unit hypercubes) to a special class of fractal sets known as string-generated Cantor sets (SCSs) [1]. An experimental mathematics approach facilitates the discovery of several closed-form results that indicate the correct formulation of the fundamental definitions of expectations over SCS fractal sets. In particular, functional equations for expectations over SCS fractal sets, supported by the underlying definitions, enable the symbolic evaluation of SCS box integrals in special cases (even-order moments or one-dimensional embeddings) and drive further developments in the theory, including the establishment of pole theorems, rationality results and the construction of a highprecision algorithm for the general numerical computation of SCS expectations.

The fundamental definition of expectations over SCS fractal sets is subsequently generalised to encompass all 'deterministic' fractal sets that can be expressed as the attractor of an iterated function system (IFS) [2]. This enables the development of generalised functional equations for expectations over IFS attractors; in particular, Proposition 5.3.4 below.

Let $\mathcal{F}=\left\{X ; f_{1}, f_{2}, \ldots, f_{m}\right\}$ be a contractive IFS with attractor $A \in \mathcal{H}(X)$. Then the expectation for a complex-valued function $F: X^{n} \rightarrow \mathbb{C}$ satisfies the functional equation

$$
\left\langle F\left(x_{1}, x_{2}, \ldots, x_{n}\right)\right\rangle=\frac{1}{m^{n}} \sum_{j_{1}=1}^{m} \sum_{j_{2}=1}^{m} \ldots \sum_{j_{n}=1}^{m}\left\langle F\left(f_{j_{1}}\left(x_{1}\right), f_{j_{2}}\left(x_{2}\right), \ldots, f_{j_{n}}\left(x_{n}\right)\right)\right\rangle .
$$

Thesis submitted to the University of Newcastle in June 2015; degree awarded on 28 September 2015; principal supervisor Jonathan Borwein, cosupervisor Brailey Sims.

(C) 2016 Australian Mathematical Publishing Association Inc. 0004-9727/2016 \$16.00 
This functional equation permits the evaluation of even-order separation moments over attractors of affine IFSs, including such celebrated fractal sets as the von Köch snowflake and the Sierpiński triangle. More generally, Proposition 5.3.4 provides a means by which the even-order box integrals of any IFS attractor generated by means of the collage theorem in order to approximate to a digital image, such as the Barnsley fern, may be symbolically resolved.

\section{References}

[1] D. H. Bailey, J. M. Borwein, R. E. Crandall and M. G. Rose, 'Expectations on fractal sets', Appl. Math. Comput. 220 (2013), 695-721.

[2] J. M. Borwein and M. G. Rose, 'Expectations over attractors of iterated function systems', Appl. Math. Comput. (2015), to appear.

MICHAEL G. ROSE, Centre for Computer Assisted Research Mathematics and its Applications (CARMA), University of Newcastle, Callaghan, NSW 2308, Australia

e-mail: michael.gerard.rose@gmail.com 